\title{
Statistical analysis of geometrical parameters of corroded reinforcement
}

\author{
Ren Wei ${ }^{1,2}$, Yingang $\mathrm{Du}^{3}$, Liang $\mathrm{Zou}^{1,2}$ and Dawang $\mathrm{Li}^{1,2}$ \\ ${ }^{1}$ Department of Civil Engineering, Shenzhen University, Shenzhen 518060, China \\ ${ }^{2}$ Guangdong Province Key Laboratory of Durability for Marine Civil Engineering (Shenzhen University), \\ Shenzhen 518060, China \\ ${ }^{3}$ Department of Engineering and the Built Environment, Anglia Ruskin University, Chelmsford CM1 1SQ, \\ The United Kingdom \\ Email of authors: lidw@szu.edu.cn(D. Li),2150150409@email.szu.edu.cn(R.Wei), \\ yingang.du@anglia.ac.uk (Y.Du) zouliang@szu.edu.cn (L. Zou)
}

\begin{abstract}
This paper reports an experimental work to investigate the pit corrosion of steel bars in chloride contaminated concrete. A total of 50 reinforcing steel bars with a length of $250 \mathrm{~mm}$ and a diameter of $14 \mathrm{~mm}$ were embedded in 10 concrete specimens, before they were subjected to accelerated corrosion. The geometric parameters of the corroded steel bars were then examined using 3D scanning method, before the characteristics of pitting corrosion of the corroded bars were analyzed accordingly. The results show that there is a weak correlation between the maximum pit depth and the mass loss rate. However, there is a strong correlation between the pitting factor and the mass loss rate. The relationship between the pitting factor and the mass loss rate has been developed as a reciprocal function.
\end{abstract}

Keywords: 3D scanning, Corrosion, Reinforcing steel bar, Statistical analysis, Chloride, Concrete.

\section{Introduction}

The corrosion of reinforcing steel is one of the principal causes of the deterioration of reinforced concrete structures, particularly for those exposed to the marine environment. Reinforcing steel corrosion in concrete is a very complicated phenomenon. It can be affected by several factors, such as the surface treatment of reinforcing bars, chemical compositions of concrete pore solution, quality and type of concrete, and the service environment of concrete structures. Owing to the heterogeneous nature of concrete and the variation and uncertainty of service environment of concrete structure, corrosion of reinforcing steel bar in concrete usually exhibits a great randomness and irregularity [1]. The irregular and uneven distribution of corrosion along the length of a reinforcing steel bar makes it difficult to determine the residual strength of the corroded steel bar more properly [2].

The pitting corrosion of reinforcing steel bars not only reduces the cross-sectional area of reinforcement but can also cause stress localization and thus may significantly decrease the loadcarrying capacity of a reinforcing bar. Numerous studies exist in the literature on the corrosion of reinforcing steel bars in concrete to examine the damage evolution of reinforcement and its effect on the load carrying capacity of the reinforced concrete structure [3-7]. Several researchers have also investigated the temporal and spatial variability of the pitting depth of corroded steel bars and pipelines in marine environment $[8,9]$ using experimental methods. The amount of corrosion of a reinforcing bar is normally represented using the corrosion current density, which can be measured from existing concrete structures using the electrochemical method. However, it should be pointed out that the corrosion current density is only an average value of the corrosion of a reinforcing bar along its length. However, pitting corrosion is usually localized, and thus it significantly different from the average corrosion and causes the minimum cross section of a reinforcing bar. It has been reported that the maximum pitting depth in a corroded steel bar in chloride contaminated concrete is about 4-10 times the average pitting depth [10]. Since the residual load bearing resistance of a corroded steel bar depends on the minimum section rather

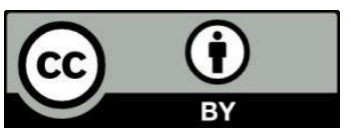

CC-BY Content from this work may be used under the terms of the Creative Commons Attribution 3.0 license. Any further distribution of this work must maintain attribution to the author(s) and the title of the work, journal citation and DOI.

Published under license in Int. J. of Computational Physics Series by NSSEL Publishing. 
than the average section of a corroded steel bar it is vital to determine the minimum cross section of a corroded steel bar. As a result, it has been suggested to use a pitting factor to calculate the maximum pitting depth that was experimentally obtained from measured pitting depths of a corroded steel bar. Tuutti reported the pitting factor could be taken as 4 to 10 for reinforcing steel bars corroded in accelerated corrosion tests [10], whereas Gonzalez et al stated the pitting factor can be taken as be 3 to 15 for reinforcing steel bars corroded in nature environment [11]. Rodriguez [12], Yu et al. [13], and Apostolopoulos [2]. Recently, Kashani et al. [14] used a 3-D scanning method to measure the 3-D surface of a corroded bar and reported the distribution of pitting depth along the circumferential and axial directions of a corroded bar. Based on the obtained data, they determined numerically the maximum pitting depth along the circumferential direction of bar section, the residual cross-sectional area, and the second moments of area of the corroded bars. In addition, the probability distribution of the residual cross-sectional area and the second moments of area of corroded bars were also settled down via the statistical analysis. However, they did not present the probability distribution of the pitting depth and its correlation with other geometric parameters of corroded bars. Hence, this paper aims to investigate the pitting corrosion of reinforcing steel bars in chloride contaminated concrete. The experimental works include the accelerated corrosion test of reinforced steel bars in concrete and the scanning of corroded steel bars using a 3-D laser scanner. The obtained 3-D images of the corroded bars are then analyzed using statistical analysis method. Unlike the work of Kashani et al. [14], this work is focused on the statistical analysis of pitting depth distribution along the length of corroded bars. Also, different from the deformed steel bars that were used in Kashani et al's tests. The plain steel bars were adopted in more severely this tests to improve the accuracy of measurement for the defined research aim.

\section{Experiment}

HPB 300 steel bar with a diameter of $14.0 \mathrm{~mm}$ was used in this t experiment. 10 concrete specimens with dimensions of $350 \times 80 \times 400 \mathrm{~mm}$ were cast. Each specimen contained five reinforcing bars with $33 \mathrm{~mm}$ concrete cover, as shown in Fig.1. The concrete mix had a targeted compressive strength of $40 \mathrm{MPa}$ at 28 days. Each reinforcing bar had a length of $400 \mathrm{~mm}$ long and was first sandblasted to remove the mill scale and rust layers on its surface. The two end parts of each bar were $75 \mathrm{~mm}$ long and were covered with epoxy first before insolated using electrical tape. The middle part of each bar was approximately $250 \mathrm{~mm}$ long and subjected to accelerated corrosion, as shown in Fig.1. The corrosion-induced mass loss of the tested reinforcing bars was in the range of $1-30 \%$, which was estimated in advance using Faraday's law of electricity and controlled through adjusting the current density or the current applied time. After the accelerated corrosion test, the corroded steel bars were carefully taken out from the specimen by breaking the concrete and then cleaned using the standard method as specified in ASTM G1-03. Afterward, the surface morphology of the corroded steel bars was measured using a 3D light scanner with the accuracy of $50 \mu \mathrm{m}$. The scanning process produced the data of three-dimensional coordinates of each point on the surface of a corroded steel bar (see Fig.2). These data were then processed using a self-compiled MATLAB program to generate various required geometrical parameters of the corroded bar, such as the pitting depth, cross-sectional area, second moments of area, and their distributions along the bar length. In the present study, the 3D measurement was taken at the intervals of both $0.5 \mathrm{~mm}$ along the bar length and $1.0^{\circ}$ around the bar sectional perimeter.

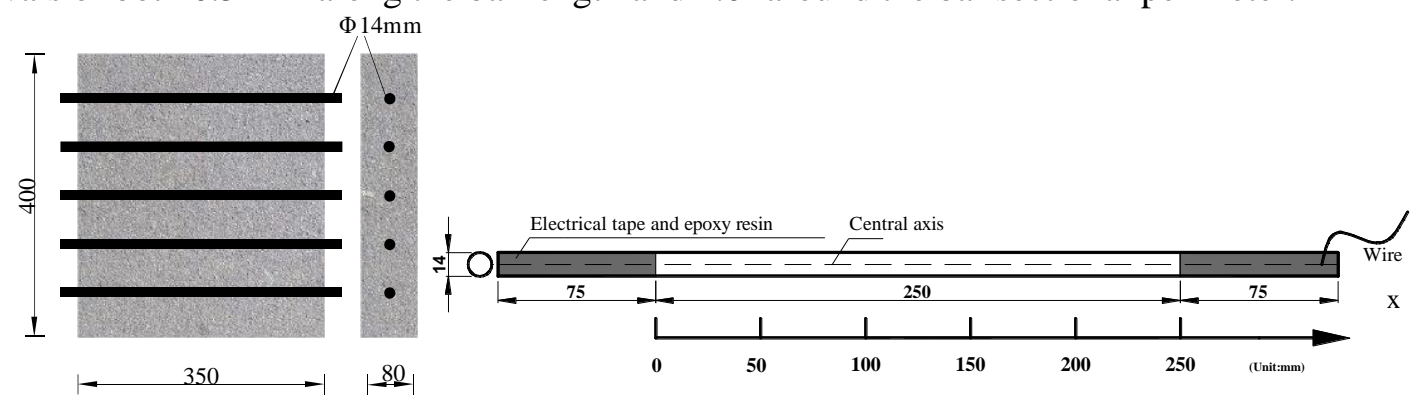

(a) Concrete specimen

(b) Reinforcing bar (unit: $\mathrm{mm}$ ).

Figure 1. Schematic of specimen design. 


\section{Results and discussion}

Owing to non-uniform corrosion, the cross-section of a corroded steel bar is no longer a circle one but more like a polygon. The $\mathrm{x}$-axis is taken along the longitudinal axis, $\mathrm{y}$ - and z-axes are the axes located on the cross-section of each steel bar, as shown in Fig.2. Let the origin of the coordinates be at the center of the original circular section of each steel bar. Then the pitting depth at a point on the surface of a corroded steel bar can be calculated as follows,

$$
P_{k}(x)=R-\sqrt{y_{k}^{2}+z_{k}^{2}}
$$

Where, $P_{k}(x)$ is the pitting depth of point $k, R$ is the radius of the original circular section of a steel bar, $y_{k}$ and $z k$ are the coordinates of the point $k$ at $\mathrm{y}$ and $\mathrm{z}$ axis, respectively.

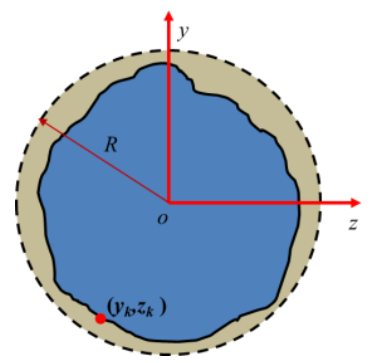

Figure 2. Definition of coordinates of a point on corroded bar surface.
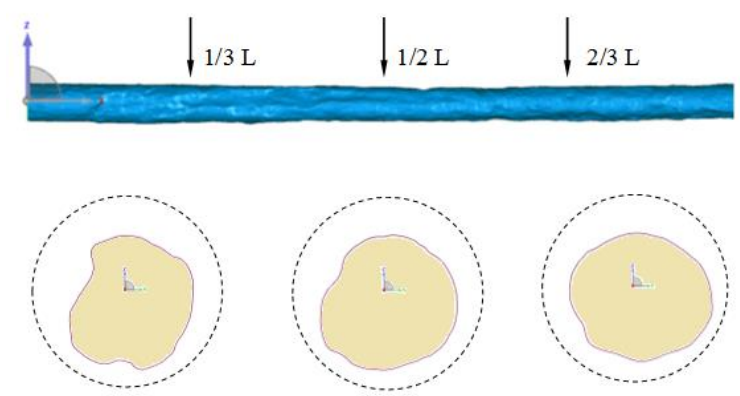

Figure 3. Residual cross-section of a corroded bar with mass loss of $15.6 \%$ at three different locations.

Fig. 3 shows the three residual cross sections of a corroded steel bar along its length. It is clear that the pit depth varied irregularly and distributed unevenly in both longitudinal and circumferential directions of the corroded steel bar. The steel bars corroded more severely on its one side where the thickness of concrete cover is minimum. The perimeter of the residual crosssection of a corroded steel bar consists of both concave and convex curves, reflecting the pitting feature. Note that the smallest diameter measured using traditional mechanical measurement methods may not reflect the pit depth at concave points and thus may not be able to give the largest pit depth in the section [15]. The largest pit depth on a section, $P_{L}(x)$, can be determined from the all measured points on that section, and calculated as follows below,

$$
P_{L}(x)=\max \left(R-\sqrt{y_{k}^{2}+z_{k}^{2}}\right)
$$

Fig.4 shows the distribution of the maximum pit depth $P_{L}(x)$ along the length of the corroded bars with four different corrosion levels in term of mass loss rates. It is obvious that the pits corrosion well developed along the length of the corroded bars and the maximum pit depth is not evenly distributed along the length of the steel bar. The largest pit depth normally increased with an increase of corrosion level. The largest $\left[P_{L}(x)\right]$ represents the maximum pit depth on the surface of a corroded bar. The longitudinal location of the max $\left[P_{L}(x)\right]$ is found different for steel bars with different corrosion levels. For example, the maximum pit depths were 2.05, 2.80, 2.93 and $3.79 \mathrm{~mm}$ that occurred at the distances of $\mathrm{x}=232.5,141.5,13.5$, and $155.5 \mathrm{~mm}$ from the left end of the steel bars with the mass loss rate of $3.6 \%, 12.1 \%, 19.7 \%$ and $31.9 \%$, respectively.

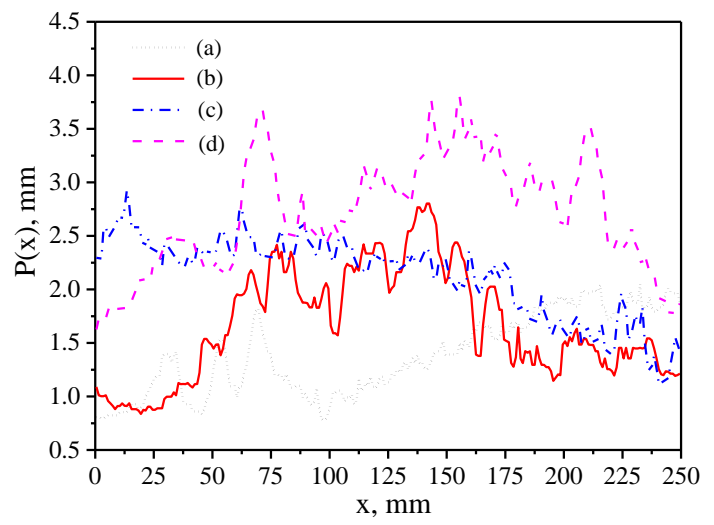

Figure 4. The distribution of the largest pit depth along length of corroded steel bars (i.e., x-axis). (a) mass loss rate $3.6 \%$, (b) mass loss rate $12.1 \%$, (c) mass loss rate $19.7 \%$, (d) mass loss rate $31.9 \%$. 
The probability distribution of $P_{L}(x)$ of the above four corroded bars is plotted in Fig.5. It is obvious that the probability distribution of $P_{L}(x)$ seems not following any single distribution pattern. For instance, the $P_{L}(x)$ distribution of the steel bars with the mass loss rates of $3.6 \%$ and $31.9 \%$ approximately exhibits the beta distribution, whereas those of the steel bars with the mass loss rates of $12.1 \%$ and $19.7 \%$ roughly follow a triangular distribution, respectively. This observation appeared different from the results of Hawn [16] and Sheikh et al. [17], who suggested a Gumball distribution for the $P_{L}(x)$, and of Strutt et al. [18], who suggested a type of I extreme value distribution for the $P_{L}(x)$. The reason for this difference is probably due to the difference between specimens used and the environmental conditions where the specimens were exposed. This further demonstrates the complexity and randomness of the corrosion of reinforcing steel in various environments.
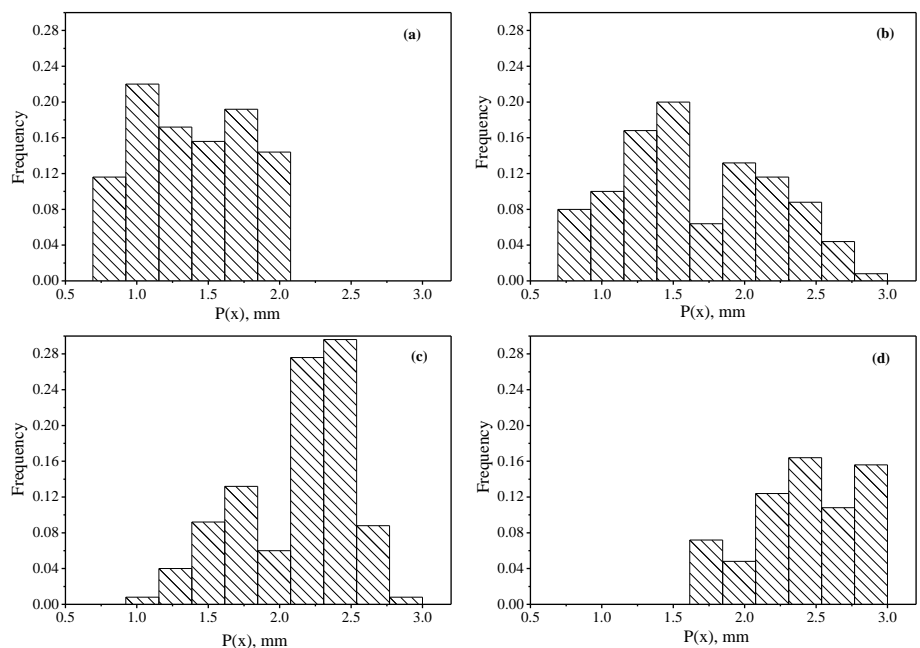

Figure 5. Histograms of pit depths of corroded bars. (a) mass loss rate $3.6 \%$, (b) mass loss rate $12.1 \%$, (c) mass loss rate $19.7 \%$, (d) mass loss rate $31.9 \%$.

Fig. 6 shows the maximum pit depth versus the mass loss rate for the 50 tested corroded bars. It is obvious that the correlation between them is rather weak, indicating that a large mass loss rate may not necessarily mean a great pit depth and vice versa. To characterize the relationship between the maximum pit depth and the mass loss rate of a corroded steel bar, one often uses the parameter called pitting factor, which is defined as follows,

$R_{p}=\frac{P_{\max }}{P_{\text {ave }}}=\frac{\max \left[P_{L}(x)\right]}{\frac{1}{L} \int_{0}^{L}\left[\frac{1}{K} \sum_{k=1}^{K} P_{k}(x)\right] d x}$

Where $P_{\max }$ is the maximum pit depth, $P_{\text {ave }}$ is the average pit depth, $L$ is the length of the corroded steel bar, and $K$ is the total number of points along the circumference of a corroded steel bar. Note that the average pit depth can also be approximately calculated using the mass loss rate as follows,

$P_{a v e}=\frac{\Delta W}{\pi D L \rho_{s}}=\frac{D \eta_{s}}{4}$

Where $\Delta W$ is the mass loss, $D$ is the original diameter of a steel bar, $\rho_{s}$ is the density of the steel bar, and $\eta_{s}$ is the mass loss rate. Fig.7 shows the plot of the pitting factor $R_{p}$ against the mass loss rate $\eta_{s}$. It is evident from the figure that there is a strong correlation between $R_{p}$ and $\eta_{s .}$ The smaller the mass loss rate, the greater the pitting factor. The relationship between both can be approximately expressed by a reciprocal function as indicated in the figure. 
Note that the mass loss rate can be approximately estimated by measuring the corrosion current. Hence, if the pitting factor $R_{p}$ is known, one can calculate the maximum pit depth by using Eq. (3) directly. Therefore, it is important to know the relationship between pitting factor and mass loss rate. From the results of the 50 tested corroded bars, it can be seen that $R_{p}$ was greater than 20 when $\eta_{s}<3 \%$ and $R_{p}$ less than 10 if $\eta_{s}>12 \%$. The reason for the pitting factor was very large if the mass loss rate is small is because when the mass loss rate is small, which causes the Pave very small, the pitting is highly localized with a large pit corrosion $P_{\max }$ developed.

According to the variation of $R_{p}$ with $\eta_{s}$ shown in Fig.7, the mass loss rate can be into divided into three different zones. The first one is the case of small corrosion case where weight loss $\eta_{s}<5 \%$ with pitting ratio $R_{p}>10$. The second is the case of medium corrosion where mass loss $5 \% \leq \eta_{s}<12 \%$ with $15>R_{p}>5$. The third is the case of severe corrosion where mass loss $\eta \mathrm{s} \geq 12 \%$ with approximately taken as 5 .

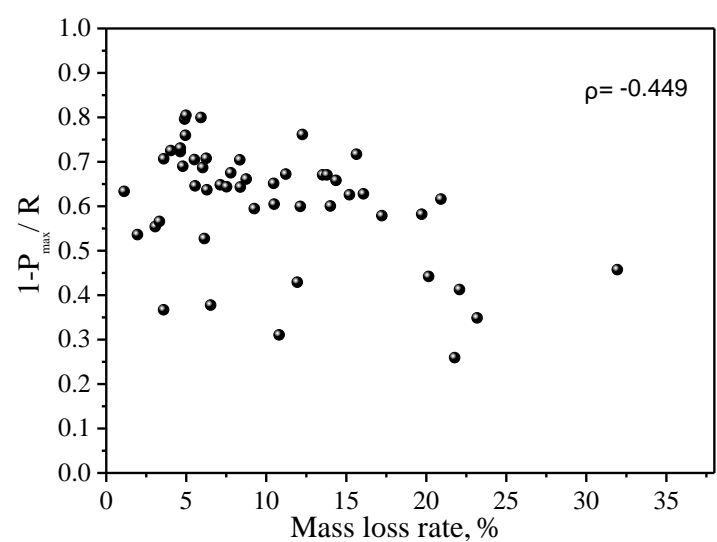

Figure 6. Correlation between the maximum pit depth and mass loss of corroded bars $(\mathrm{R}=7 \mathrm{~mm}$ is the radius of steel bar).

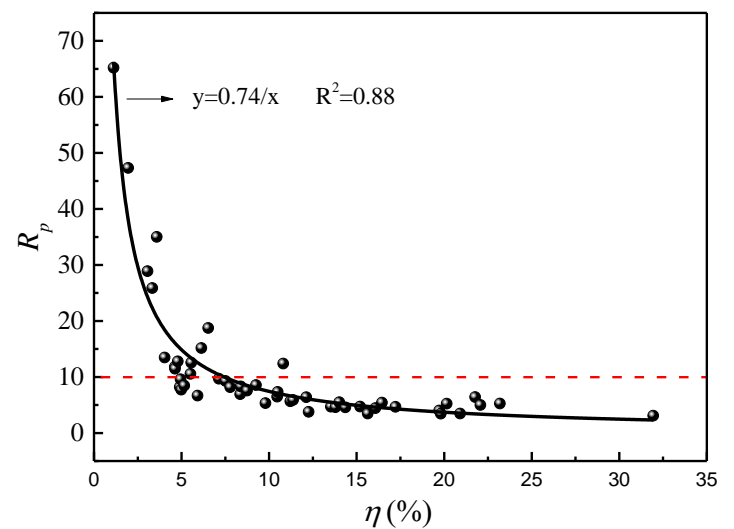

Figure 7. Relationship between pitting factor and mass loss rate

\section{Conclusion}

In this paper, the pit corrosion of reinforcing steel bars in chloride contaminated concrete has been investigated experimentally using accelerated corrosion tests and 3-D scanning technique. Based on the 3-D images of 50 corroded steel bars the pit depth and its corresponding probability distribution along the length of each corroded bar were analyzed using statistical analysis methods. From the analysis, it is easily know that the pit depth of a corroded bar varies irregularly and distributes unevenly in both circumferential and longitudinal directions of a corroded bar. It's distribution along the length of a bar seems not fitting for any single existing probability modal. And the maximum depth of corrosion pit increases with an increase in corrosion level or mass loss. However, the correlation between the values of the maximum pit depths and the mass loss rate seems less strong. Furthermore, it can be found that there is a strong correlation between the pitting factor and the mass loss rate. The smaller the mass loss rate, the greater the pitting factor. The relationship between them can be approximately expressed using the recommended reciprocal function in the paper. For the case of small corrosion case where weight loss $\eta_{s}<5 \%$, the pitting ratio $R_{p}$ is larger than 10 . For the case of medium corrosion where mass loss $5 \% \leq \eta_{s}$ $<12 \%$, the pitting ratio $R_{p}$ ranges from 5 to 15 . For the case of severe corrosion where mass loss $\eta_{s} \geq 12 \%$, the pitting ratio $R_{p}$ can be approximately taken as 5 .

Acknowledgement - The authors at Shenzhen University would like to acknowledge the financial support provided by the National Natural Science Foundation of China (Grants Nos. 51520105012, 51278303), the Key Project of Department of Education of Guangdong Province

( Grants No.2014KZDXM051) and Shenzhen University. 


\section{References:}

[1] S. Muthulingam, B.N. Rao. Non-uniform corrosion states of rebar in concrete under chloride environment. CORROS SCI. 93 (2015), 267-282.

[2] C.A. Apostolopoulos, S. Demis, V.G. Papadakis. Chloride-induced corrosion of steel reinforcement - Mechanical performance and pit depth analysis. CONSTR BUILD MATER. 38 (2013), 139-146.

[3] C.A. Apostolopoulos, V.G. Papadakis. Consequences of steel corrosion on the ductility properties of reinforcement bar. CONSTR BUILD MATER. 22 (2008), 2316-2324.

[4] A.A. Almusallam. Effect of degree of corrosion on the properties of reinforcing steel bars. CONSTR BUILD MATER. 15 (2001), 361-368.

[5] W.P. Zhang, H.C. Dai, X.L. Gu, S.N. Wu. Effects of Corrosion Pits on Mechanical Properties of Corroded Steel Bars. Workshop on Biennial International Conference o... (2015).

[6] M.G. Stewart, A. Al-Harthy. Pitting corrosion and structural reliability of corroding RC structures: Experimental data and probabilistic analysis. RELIAB ENG SYST SAFE. 93 (2008), 373-382.

[7] L.Y. Li, C.L. Page. Finite element modeling of chloride removal from concrete by an electrochemical method. CORROS SCI. 42 (2000), 2145-2165.

[8] F. Caleyo, J.C. Velázquez, A. Valor, J.M. Hallen. Probability distribution of pitting corrosion depth and rate in underground pipelines: A Monte Carlo study. CORROS SCI. 51 (2009), 19251934.

[9] W. Zhang, B. Zhou, X. Gu, H. Dai. Probability Distribution Model for Cross-Sectional Area of Corroded Reinforcing Steel Bars. J MATER CIVIL ENG (2014).

[10] K. Tuutti. Corrosion of steel in concrete. (1982).

[11] J.A. González, C. Andrade, C. Alonso, S. Feliu. Comparison of rates of general corrosion and maximum pitting penetration on concrete embedded steel reinforcement. Cement \& Concrete Research. 25 (1995), 257-264.

[12] J. Rodriguez, L.M. Ortega, J. Casal. Load carrying capacity of concrete structures with corroded reinforcement. CONSTR BUILD MATER. 11 (1997), 239-248.

[13] L. Yu, R. François, V.H. Dang, V. L Hostis, R. Gagné. Distribution of corrosion and pitting factor of steel in corroded RC beams. CONSTR BUILD MATER. 95 (2015), 384-392.

[14] M.M. Kashani, A.J. Crewe, N.A. Alexander. Use of a 3D optical measurement technique for stochastic corrosion pattern analysis of reinforcing bars subjected to accelerated corrosion. CORROS SCI. 73 (2013), 208-221.

[15] D. Li, R. Wei, Y. Du, X. Guan, M. Zhou. Measurement methods of geometrical parameters and amount of corrosion of steel bar. Construction \& Building Materials. 154 (2017), 921-927.

[16] D.E. Hawn. Extreme value prediction of maximum pits on pipelines. Mater, Performance, (United States). 16 (1977), 29-32.

[17] A.K. Sheikh, J.K. Boah, D.A. Hansen. Statistical Modeling of Pitting Corrosion and Pipeline Reliability. Corrosion -Houston Tx-. 46:3 (2012), 190-197.

[18] J.E. Strutt, J.R. Nicholls, B. Barbier. The prediction of corrosion by statistical analysis of corrosion profiles. CORROS SCI. 25 (1985), 305-315. 